\title{
Sustainable Communication
}

\author{
Wim JL Elving* \\ Professor of Applied Sciences, Centre of Expertise Energy, Hanze University of Applied Sciences, the Netherlands
}

Submission: October 29, 2020; Published: November 11, 2020

*Corresponding author: Wim JL Elving, Professor of Applied Sciences, EnTranCe, Centre of Expertise Energy, Hanze University of Applied Sciences, Nijenborgh 6, 9747 AG Groningen, the Netherlands

\section{Abstract}

In this mini-review I will present a practical way how communication relates to sustainability and how communication can be used in various stages to increase sustainability and to accelerate the energy transition. The model, from A to Sustainability, details the various forms of communication, from information provision, to persuasion to dialogue.

Keywords: Communication; Urgency; Awareness; Action; Dialogue

\section{Introduction}

Communication is a container term, that includes information, persuasion, dialogue and many other terms. Many definitions of communication have been given, most have is the process of passing information and understanding from one person to another. In simple words it is a process of transmitting and sharing ideas, opinions, facts, values etcetera from one person to another or one organization to another. Communication and behaviour are interlinked and behaviour cannot change without communication. The basis of change is information, preferable high quality, scientific based information, is the basis for the Energy transition and the dialogue for our future society. Information needs to be correct, on time, and needs to include the main questions and worries of the receivers [1].

Communication becomes more and more included in projects to increase sustainability and to accelerate the energy transitions. We need communication to explain various new techniques, risks involved with these, but also in finding public support for innovations and the transition from old habits. Energy is a technical term and extensive defined. As humans we need, just as other living organisms' energy to stay alive, obtained by food and water. Our society needs energy for functioning, in which we differ between energy sources obtained by fossil fuels, nuclear fuel or renewable sources. In order to prevent further rise of temperatures and to prevent the climate crisis we need to limit the amount of greenhouse gasses and make the transition from fossil fuels to renewable energy.

Though differences exist worldwide, the common view is that all fossil fuels lead to an increase of levels of carbon or greenhouse gasses in the atmosphere and that by reducing greenhouse gases we will limit the temperature rise and climate crisis. The energy transition from fossil fuels to renewable energy is a major challenge for society. We are currently using fossil fuels for transport, for heating, for production and all aspects of modern life and for our economy. The internet gave us the opportunities for global trade, we can easily buy items from all over the world, but transport of these items, most of the times is done with fossil fuels. How can we limit our use of fossil fuels without limiting prosperity in developing countries?

In a transition, like the energy transition, it is important to repeat the main reason for the change, sometimes forgotten in the complexity of the change or transition. In our case: we need to limit our carbon footprint to limit the consequences of climate change is the phrase we need to repeat. Sustainability communication is in our view the communication needed to persuade, convince and inform publics to adopt a sustainable lifestyle [1]. We need to apply theoretical insights in communication and explore applied research in accelerating the energy transition and establish the sustainable society. In applying the lessons from organizational, corporate communication and PR and marketing communication for these goals we help society at large and individuals in this. We use our research model, from A to Sustainability to explain the steps needed (see figure 1).

As been listed above, the why of the energy transition is that we need to stop climate change, for instance by limiting our personal carbon footprint. There are still a lot of climate sceptics, which numbers seem to be higher among politicians and lawmakers who do not believe climate change is real, despite the fact that IPCC 
supported by almost $100 \%$ of climate scientists did proof that the increase of levels of carbon dioxide $\left(\mathrm{CO}_{2}\right)$ / greenhouse gasses is coming from human activity and especially the burning of fossil fuels.

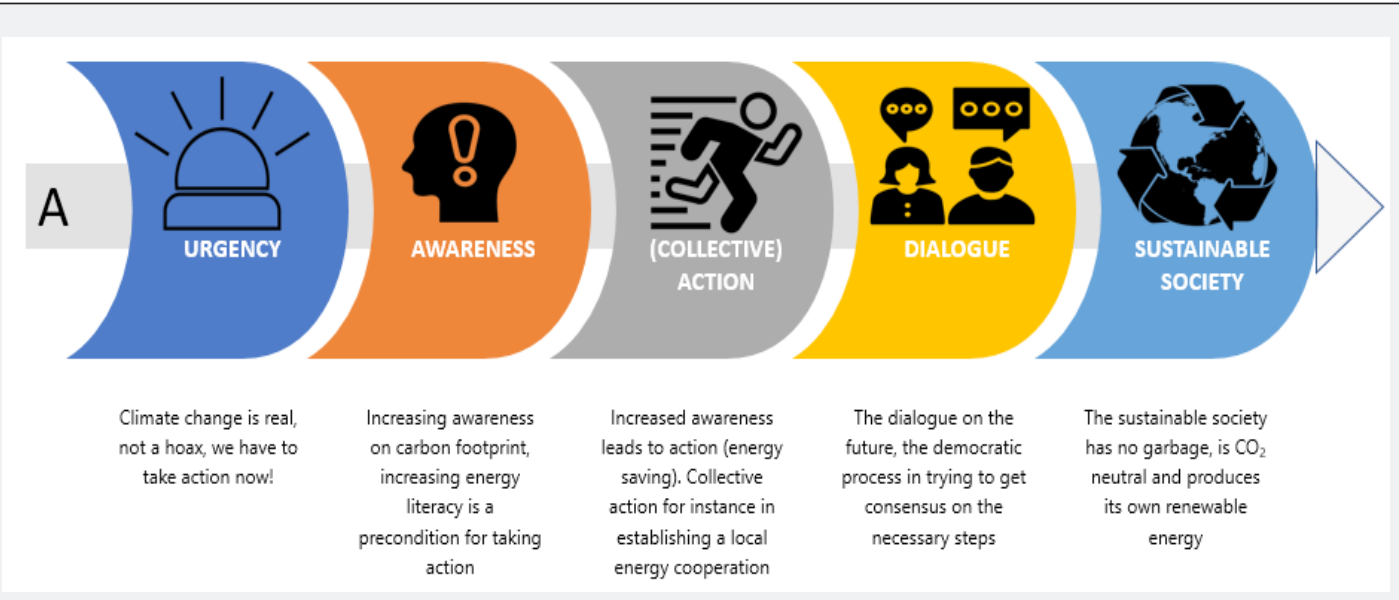

Figure 1: Our process model: from A to Sustainability.

Awareness is defined as the ability to directly know and perceive, to feel, or to be cognizant of events [2] and seems to be one of the crucial elements in changing behavior [1]. More broadly, awareness is the state of being conscious of something. Another definition describes it as a state wherein a subject is aware of some information when that information is directly available to bring to bear in the direction of a wide range of behavioral processes. The concept is often synonymous to consciousness and is also understood as being consciousness itself [3]. Awareness is crucial regarding the energy transition and the establishment of a sustainable society. If you are unaware of the damages to the environment or climate your actions are doing, you will not alter your behavior.

If awareness is a fact and individuals are willing to take steps to reduce their own ecological footprint, it is important that sufficient information is available about possible measures. This is a question of the transfer of information and knowledge, and therefore of information and public education.

Collective action, on the other hand, is somewhat more complex, because collective action presupposes a consensus on the measures to be taken. Collective action is also often organized by the (local) government. Public education is of importance here, because it is needed to act on the basis of the right information and the conviction that the sender of this information can be trusted and is credible. The unilateral imposition of measures is often counterproductive; it is important to involve people as much as possible in measures, which is of course a complex task.

\section{Dialogue}

The most crucial element in our model is dialogue. Dialogue in nature is the richest way of exchanging information and views. It is important to distinguish the dialogue from the conversation.
The dialogue is a spoken or written conversation between two or more people, but it goes beyond a conversation. The Socratic method [4] is still used in our time to find out through dialogue how a problem could be made up. In a dialogue, clarity is obtained about the different opinions and feelings, about motivations and presuppositions. Dialogue is often presented as an ideal way to achieve results, but in fact the aim of the dialogue is, at most, to increase mutual understanding. We must not, therefore, use dialogue in an inappropriate way. There are many situations in which dialogue is not desirable or not possible. In those situations, it is better to limit yourself to giving information. Dialogue can be used in engaging society. How can we engage with the people from a specific region to develop ways for renewable energy, limit waste, recycle and for the circular economy is the real challenge in creating the sustainable society? Engagement is often used in a romantic relationship, and within the challenges ahead of us all, we need to connect with all members of society. We only have very little time to stop climate change.

\section{Discussion}

In this short paper I have tried to explain sustainable communication in a practical way, by addressing different forms of communication, from informing to persuasion, from repeating (and repeating if necessary) the message about the urgency to climate sceptics, and dialogue and engagement. Communication is central in changing behaviors, habits and cultures, and central in accelerating the energy transition and establishing the sustainable society.

\section{References}

1. Elving WJL (2019) Duurzaamheidscommunicatie. Inaugural speech. Groningen: Hanze University of Applied Sciences.

2. Chalmers D (1997) The Conscious Mind: In Search of a Fundamental Theory. Oxford: Oxford University Press, USA, p. 225. 
3. Hussain A, Aleksander I, Smith L, Barros A, Chrisley R, et al. (2009) Brain Inspired Cognitive Systems. New York: Springer Science + Business Media, p. 298.
4. Altorf HM (2016) Dialogue and discussion: Reflection on a Socratic method. Arts and Humanities in Higher Education 18(1): 60-75.

DOI:10.19080/IJESNR.2020.26.556190 